\title{
The grand challenges to cellular and infection microbiology
}

\author{
Yousef Abu Kwaik ${ }^{1,2 *}$ \\ 1 Department of Microbiology and Immunology, College of Medicine, University of Louisville, Louisville, KY, USA \\ 2 Department of Biology, University of Louisville, Louisville, KY, USA \\ ${ }^{*}$ Correspondence: abukwaik@louisville.edu
}

Almost one and a half centuries have passed since Robert Koch and Thomas Burrill made the seminal discovery that bacteria are responsible for mammalian and plant diseases. Shortly after, it was found that parasites, fungi, and viruses are also etiological agents of diseases in multi-cellular eukaryotes. As we roam the earth, we realize that we are outnumbered by the microbial community; that each of us has a microbiota which exceeds in numbers our own cells; and that the microbiota are essential components of our development and health. Another milestone was set by Robin Warren and Barry Marshall who discovered that a bacterium is responsible for gastric ulcer and gastric carcinoma, which earned them the 2005 Nobel Prize in Medicine, and changed our views and perspectives of other diseases. It opened the door to the possibility that microorganisms may be responsible for many other diseases of unknown etiology, such as Alzheimer's, gastrointestinal diseases of unknown etiology, and cardiovascular diseases.

The past few decades have witnessed a quantum leap in our knowledge of microbe-host interactions. This has been fueled by the discovery of the polymerase chain reaction (PCR), the determination of a large number of genomic sequences, advancements in cell biology and immunology, and the development of various high throughput arrays for transcriptome and proteome analyses. As genomic sequencing has become more feasible, our understanding of pathogenic evolution and patho-adaptation to the host made tremendous strides. It is becoming clear that several of the specialized secretion systems are evolutionarily related to phages, and that phages may be an even more important driving force in the evolution of virulence than previously appreciated. In addition, novel approaches have been utilized to identify a myriad of microbial effectors that are delivered to targets in the host cell by elaborate type III-VII translocation devices leading to modulation of various cellular processes (Holland, 2010). The advances in genetic manipulations of eukaryotic cells, along with the development of various genetically defined animal models, have had and will continue to have tremendous impact on our knowledge of host-microbe interactions. Recent years have witnessed major progress in our understanding of innate immunity to infection, but we need to further expand our understanding of adaptive immunity, which has been lagging. Still, our progress in understanding host-microbe interactions has been nothing less than remarkable. Having said that, it is also quite fascinating how little this has helped in generating new vaccines for various infectious microbes or developing new therapies against multi-drug resistant bacteria. It is also astonishing how few viral infections have effective therapeutic agents or vaccines. This dichotomy clearly indicates that, although our excitement and hyperventilation about the new advances is well justified, we are only grasping the tip of the iceberg of host-microbe interactions, and our combined excitement and denial of this shallow understanding of hostmicrobe interactions keeps convincing us otherwise! There are numerous questions to be answered, as we dig deeper using the molecular reductionist approach, where the focus has switched from the big picture of the disease to the study of microbe-host interaction at the molecular level.

Acknowledging these limitations, the "Grand Challenges" are put forth to encourage coordinated efforts to accomplish high impact goals that we currently think are unachievable. Setting targets for ourselves is essential for our ultimate aspiration of generating knowledge and platforms integrated across many biomedical disciplines that will benefit public health throughout the world. The challenges that Frontiers in Cellular and Infection Microbiology faces are highly interconnected, and overlap with those of many other biomedical disciplines.

\section{HOST-MICROBE INTERACTIONS}

Our recent advances led us to a more indepth understanding of host-microbe interactions, and have fostered dominance of the reductionist approach. Molecular reductionism is an extremely powerful approach, but to be strengthened and expanded, it must be combined with a focused approach to capture the big picture of diseases. We face major challenges to develop more appropriate animal models that more reliably predict the course and outcome of human infectious diseases. As we develop these, we must keep in mind that the models we use are animal models, which may not be extrapolated accurately to the human host. The big interdisciplinary challenge to biomedical sciences is to create computational virtual human and mouse model systems that are experiment-based, high-precision, multiscaled, and comprehensive (Kitano, 2010). Virtual human and animal model systems will be the "tool" that is needed for many biomedical and non-biomedical disciplines, including systems biology, biophysics, and infection and immunity. The development of these virtual models will allow simulation and prediction of the consequences of host-microbe interactions, and translation of these interactions from a molecularbased reductionist approach to a host-based knowledge of the big picture of diseases. The consolidation and integration of isolated novel and seminal findings is as important as the findings themselves, because without this integration the grand challenge will not be met. Taken together, one of our major challenges is to combine the molecular reductionist approach with the big picture of diseases, and analyze molecular interactions in living cells and in animal models at the ultra-structural level.

\section{SELECT AGENTS}

As our understanding of molecular and cellular aspects of host-microbe interactions has advanced, so has the potential misuse of microorganisms to destroy communities and societies. This has generated interest 
in developing therapeutic and preventive measures to select agents. It would be wise to first acquire the basic knowledge of the biology and pathogenesis of the select agents; otherwise, our efforts to develop rapid therapeutic and preventive measures may be misguided.

\section{EVOLUTION OF FUNCTIONAL AND STRUCTURAL MIMICRY OF EUKARYOTIC PROTEINS IN PATHOGENIC MICROORGANISMS}

It is becoming evident that many pathogenic microorganisms have co-evolved in the environment with other prokaryotic and primitive eukaryotic organisms. One of the grand challenges is to continue the study of the evolutionary aspects of pathogenic microorganisms and to identify and develop as models lower eukaryotic hosts that likely contributed to the evolution of pathogenicity in many microorganisms. The development of these less complex model hosts will require detailed studies to understand the biology of these hosts. Deciphering the interaction of pathogenic microorganisms with their environmental primitive hosts should thus be an integral component of our studies with the multicellular mammalian and plant hosts. A myriad of effectors injected by microbial pathogens into the host cell seem to have eukaryotic structural and functional similarities, such as Ankyrin repeat-containing proteins, GTPase, SNAREs, and F-box proteins (Price et al., 2009; Al-Khodor et al., 2010). Therefore, understanding the genetic acquisition of these eukaryotic-like proteins will be essential for our understanding of pathogenic evolution and host adaptation of microbes.

For example, the interaction of Legionella with protozoa and other primitive eukaryotes has most likely been a major factor in evolving its ability to infect humans (Molmeret et al., 2005). Co-evolution with protozoa has shaped pathogenic evolution of Legionella to exploit evolutionarily conserved eukaryotic processes that have facilitated pulmonary infection of the human host. Examples of these conserved eukaryotic processes exploited by Legionella in evolutionarily distant hosts are the polyubiquitination (Price et al., 2009) and farnesylation machineries (Price et al., 2010). Interestingly, exploitation of both eukaryotic machineries is essential for intracellular bacterial proliferation within mammalian macrophages and amoeba, and for intrapulmonary proliferation of Legionella in the mouse model of the disease. Remarkably, both eukaryotic machineries are exploited by the same injected effector, Ankyrin B, most of which is composed of four eukaryotic motifs: two ankyrin repeats, an F-box domain, and a $C$-terminus "CaaX" farnesylation motif (Price et al., 2009, 2010). Studies on genetic acquisition and evolution of this and other microbial eukaryotic-like proteins in bacterial effectors that are specifically recognized by bacterial chaperones and injected into the host cell by specialized type III-VII secretion machineries should yield interesting insights into host-microbe adaptation and pathogenic evolution. As advances in structural biology and other disciplines continue, resolving structural aspects of the microbial effectors and their injections through the translocation apparatuses, along with the sequential molecular interactions during the injection process, will be a challenge. However, we should be able to tackle that challenge in the near future, particularly with continued advances in related disciplines.

For injected bacterial effectors, deciphering the evolution of structural and functional mimicry of eukaryotic posttranslational modification motifs, such as the Fic motif for AMPylation and the CaaX motif for farnesylation (Broberg and Orth, 2010; Price et al., 2010), will require further advances in our bioinformatical analyses. Our study of the role of inter-kingdom horizontal gene transfer and convergent evolution in the acquisition of eukaryotic-like proteins and motifs requires improvements in computational models to decipher the mechanism of this genetic evolution. The recent achievements of a functional synthetic microbial genome by Craig Venter and his co-workers will soon become a standard tool that will speed up our progress and understanding of microbial evolution as well as host-microbe interaction.

Although, our approaches to identify injected bacterial effectors have been innovative, the signals that trigger translocation of the effectors are still unknown. We do understand that pathogens sense their attachment to the host cell to trigger translocation, but the process is most likely more complex at the molecular level and requires cross-talk between the host cell and the microbe. Understanding the molecular details involved in this cross-talk will continue to challenge us, but our innovations will overcome every obstacle that may hinder our progress in seeking this and other knowledge. Understanding the molecular details that trigger translocation of effectors will facilitate the development of therapeutic and preventive measures to target the translocation machineries, and block the signals that trigger them. This may have the potential of a common countermeasure against various pathogens.

\section{GASTROINTESTINAL MICROBIOTA}

Understanding how the magic balance between the gastrointestinal microbiota influences human health, disease and the variability between individuals will be one of the most challenging tasks, which will go far beyond understanding mechanisms of microbial pathogenesis in infectious diseases (Garrett et al., 2010). We are challenged to understand the imbalance of the gut microbiota (dysbiosis) and the detailed nature of this imbalance, which is believed to be the etiology of many modern diseases that are invading the developed world at lightning speed. These diseases include, but are not limited to, asthma, food allergies, Crohn's disease and irritable bowel syndrome (Huffnagle, 2010). The consequences of short and long term use of antibiotics, and their short and long term effects on the gut microbiota, require detailed studies. The challenges to decipher the complexity of the gut microbiota are enormous, considering individual variations, which could result in significant consequences that may be underestimated. There is no doubt that the next few decades will reveal remarkable findings related to the skin, oral, vaginal, nasal, and gut microbiota, which will benefit public health tremendously. The use of probiotics, prebiotics, and neutraceuticals has become widespread without any in-depth understanding of their use. The high complexity of the gut microbiota will continue to challenge us for decades, since most of them are uncultured.

\section{MOVING UNCULTURED ORGANISMS TO THE CULTURED COMMUNITY}

Another grand challenge is to develop culture media to add the uncultured microbiota and the obligate intracellular bacteria to the cultured community of microbes. The recent development of a growth medium for 
Coxiella by Robert Heinzen and colleagues (Omsland et al., 2009) is a beautiful example of the combination of post-genomic analyses, basic bacterial metabolism and physiology to formulate the composition of the medium to grow, what was known as an obligate intracellular bacterium. This is a seminal achievement that will definitely enhance the development of genetic tools to dissect various aspects of microbe-host interaction, and it certainly provides many glimpses of hope that we will succeed in culturing other obligate intracellular as well as extracellular yet uncultured bacteria.

\section{WIRELESS MICROBIAL COMMUNICATION}

The discovery of wireless bacterial social communication through quorum sensing in the marine bacterium Vibrio fischeri, which started in the 1960s, has led to a new paradigm in infectious diseases and hostmicrobe interactions (Miller and Bassler, 2001). The identification of the chemical structure of the inducer by Oppenheimer and colleagues (Eberhard et al., 1981) has opened wide the doors to understand the biochemical and genetic bases of microbial communication. These seminal discoveries, pioneered by many experts including Barbara Iglewski, Peter Greenberg, and Bonnie Bassler, have changed our thoughts and perspectives of infectious diseases and microbial ecology. The cross-species communication through quorum sensing during infections of the oral, GI, or vaginal tract will continue to evolve, but understanding this cross-species communication is challenged by the complexity of the respective microbiota. Understanding the complexity of these systems will take time, and is tightly linked to developments in other related disciplines.

\section{MOVING FORWARD}

Undoubtedly, biomedical sciences must continue to expand and strengthen their interdisciplinary approach to decipher biological processes. Since the grand challenges that many biomedical disciplines face are interlinked and unmistakably overlap, developments in other fields, such as synthetic chemistry and biophysics of imaging, will continue to benefit and advance our progress in cellular and infection microbiology and host-microbe interaction.

The grand challenges encountered by the post-omic era is to integrate the molecular reductionist approach and the powerful information obtained from in vitro studies into a comprehensive understanding of the big picture of the disease in vivo. The time is ripe to reinvigorate Koch's postulate, and integrate it into our reductionist molecular approach to provide a more in-depth knowledge of disease in the host.

To deal with the tremendous advances in research and its global expansion, innovations in the communication of research findings are continuing to evolve. While other journals think open access is the future for scientific publishing, the Frontiers Research Foundation believes that it is needed now, which will lead us to a more innovative future in scientific publishing and, therefore, the foundation has positioned itself at the forefront of innovation of academic publishing.

\section{ACKNOWLEDGMENTS}

Research work in the Yousef Abu Kwaik lab is supported by Public Health Service Awards R01AI43965 and R01AI069321 from NIAID at the National Institute of Health, and by the commonwealth of Kentucky Research Challenge Trust Fund. The author thanks John Leong and Sanke Jones for proofreading and helpful comments on this article.

\section{REFERENCES}

Al-Khodor, S., Price, C. T., Kalia, A., and Abu Kwaik, Y. (2010). Ankyrin-repeat containing proteins of microbes: a conserved structure with functional diversity. Trends Microbiol.18, 132-139.
Broberg, C. A., and Orth, K. (2010). Tipping the balance by manipulating post-translational modifications. Curr. Opin. Microbiol.13, 34-40.

Eberhard, A., Burlingame, A. L., Eberhard, C., Kenyon, G. L., Nealson, K. H., and Oppenheimer, N. J. (1981). Structural identification of autoinducer of photobacterium fischeri luciferase. Biochemistry 20, 2444-2449.

Garrett, W. S., Gordon, J. I., and Glimcher, L. H. (2010). Homeostasis and inflammation in the intestine. Cell 140, 859-870.

Holland, I. B. (2010). The extraordinary diversity of bacterial protein secretion mechanisms. Methods Mol. Biol. 619, 1-20.

Huffnagle, G. B. (2010). The microbiota and allergies/ asthma. PLoS Pathog. 6, e1000549. doi: 10.1371/journal.ppat.1000549.

Kitano, H. (2010). Grand challenges is systems physiology. Front. Syst. Physiol. 1, 1-3.

Miller, M. B., and Bassler, B. L. (2001). Quorum sensing in bacteria. Annu. Rev. Microbiol. 55, 165-199.

Molmeret, M., Horn, M., Wagner, M., Santic, M., and Abu Kwaik. (2005). Amoebae as training grounds for intracellular bacterial pathogens. Appl. Environ. Microbiol. 71, 20-28.

Omsland, A., Dale Howe, D., Fischer, E. R., Virtanevac, K., Sturdevant, D. E., Porcella, S. F., and Heinzen, R. (2009). Host cell-free growth of the Q fever bacterium Coxiella burnetii. Proc. Natl. Acad. Sci. U.S.A. 11, 4430-4434.

Price, C. T., Al-Khodor, S., Al-Quadan, T., Kalia, A., and Abu Kwaik, Y.(2009). Molecular Mimicry by an F-box effector of Legionella pneumophila hijacks a conserved polyubiquitination machinery within macrophages and protozoa. PLoS Pathog. 12, e1000704. doi: 10.1371/journal.ppat.1000704.

Price, C. T., Al-Quadan, T., Santic, M., and Abu Kwaik, Y. (2010). Exploitation of conserved eukaryotic host cell farnesylation machinery by an F-box effector of Legionella pneumophila. J. Exp. Med. 207, 1713-1726.

Received: 01 June 2010; accepted: 07 June 2010; published online: 21 September 2010.

Citation: Kwaik YA (2010) The grand challenges to cellular and infection microbiology. Front. Microbio. 1:3. doi: 10.3389/fmicb.2010.00003

This article was submitted to Frontiers in Cellular and Infection Microbiology, a specialty of Frontiers in Microbiology.

Copyright (C) 2010 Kwaik. This is an open-access article subject to an exclusive license agreement between the authors and the Frontiers Research Foundation, which permits unrestricted use, distribution, and reproduction in any medium, provided the original authors and source are credited. 